(2) Open Access Full Text Article

\title{
Spectrum of bactericidal action of
} amylmetacresol/2,4-dichlorobenzyl alcohol lozenges against oropharyngeal organisms implicated in pharyngitis

This article was published in the following Dove Press journal:

International Journal of General Medicine

\section{Derek Matthews \\ Robert Atkinson \\ Adrian Shephard}

Reckitt Benckiser Healthcare International Ltd, Slough, Berkshire, UK
Correspondence: Robert Atkinson Reckitt Benckiser Healthcare International Ltd, 103-105 Bath Road,

Slough, Berkshire SLI 3UH, UK

Tel +44 I48 2583969

Fax +44 1753217899

Email robert.atkinson@rb.com
Purpose: Pharyngitis is commonly caused by a self-limiting upper respiratory tract infection (URTI) and symptoms typically include sore throat. Antibiotics are often inappropriately used for the treatment of pharyngitis, which can contribute to antimicrobial resistance, therefore nonantibiotic treatments which have broad antiseptic effects may be more appropriate. Amylmetacresol (AMC) and 2,4-dichlorobenzyl alcohol (DCBA) are present in some antiseptic lozenges and have established benefits in providing symptomatic relief and some in vitro antiviral action.

Methods: Seven bacterial species associated with pharyngitis, namely Streptococcus pyogenes, Fusobacterium necrophorum, Streptococcus dysgalactiae subspecies equisimilis, Moraxella catarrhalis, Haemophilus influenza, Arcanobacterium haemolyticum and Staphylococcus aureus, were exposed to an AMC/DCBA lozenge dissolved in artificial saliva. In vitro bactericidal activity was measured as a log reduction in colony-forming units (CFUs).

Results: Bactericidal activity was recorded against all organisms after 1 minute. Greater than 3 $\log _{10}$ reductions in CFUs were observed at 1 minute for $S$. pyogenes $\left(\log _{10}\right.$ reduction CFU/mL \pm $\mathrm{SD}, 5.7 \pm 0.1)$, H. influenza (6.1 \pm 0.1$)$, A. haemolyticum (6.5 \pm 0.0$)$ and F. necrophorum $(6.5 \pm 0.0)$, at 5 minutes for $S$. dysgalactiae $(6.3 \pm 0.0)$ and M. catarrhalis $(5.0 \pm 0.9)$ and at 10 minutes for S. aureus $(3.5 \pm 0.1)$.

Conclusion: An AMC/DCBA lozenge demonstrated a greater than $99.9 \%$ reduction in CFUs against all tested species within 10 minutes, which is consistent with the time a lozenge remains in the mouth. Patients with uncomplicated bacterial pharyngitis may benefit from the antibacterial action of antiseptic AMC/DCBA lozenges. Furthermore, AMC/DCBA lozenges may be more relevant and appropriate than antibiotics for pharyngitis associated with a self-limiting viral URTI.

Keywords: pharyngitis, bacterial infections, antibacterial agents, Streptococcus, sore throat

\section{Plain language summary}

Pharyngitis is a common condition. It can last several days and is usually the result of self-limiting viral infections, such as the common cold, although occasionally, pharyngitis can be caused by a bacterial infection. The most commonly reported symptom is sore throat. Antibiotics do not work against the viruses that in most cases cause pharyngitis but are often prescribed anyway. This contributes to antimicrobial resistance, where bacteria become immune to antibiotics and treatment for infections becomes difficult. Alternative treatments could help reduce inappropriate prescriptions of antibiotics for pharyngitis, and previous studies have demonstrated the antiviral and pain-relieving qualities of some antiseptic lozenges. The authors conducted a laboratory- 
based study to assess the ability of antiseptic lozenges to kill a broad range of bacteria known to cause pharyngitis. They found that, when lozenges containing two antiseptic ingredients were dissolved in a solution similar to human saliva, the mixture killed $99.9 \%$ of all pharyngitis-associated bacteria that were tested within 10 minutes. These results suggest that patients with uncomplicated bacterial pharyngitis may benefit from the antibacterial and painrelieving action of antiseptic lozenges, including those taking antibiotics. Additionally, antiseptic lozenges may be more relevant and appropriate than antibiotics for pharyngitis of a viral origin.

\section{Introduction}

Pharyngitis is associated with inflammation of the pharynx ${ }^{1}$ and is one of the most common reasons patients seek health care professional advice. ${ }^{2}$ Acute pharyngitis is predominantly caused by a viral upper respiratory tract infection such as the common cold ${ }^{3,4}$ and is usually self-limiting with symptoms, such as sore throat, lasting $\sim 3-7$ days. ${ }^{5}$ Despite this, antibiotics are still frequently inappropriately used for the treatment of pharyngitis even though patients consulting their doctor are often primarily seeking reassurance and symptomatic relief. ${ }^{6,7}$ Antibiotics are ineffective against the viruses that cause $\sim 90 \%$ of cases, do not offer symptomatic relief and inappropriate antibiotic prescription can contribute to antimicrobial resistance, which is a serious threat to global public health. ${ }^{8}$ Consequently, there is a need for non-antibiotic treatments, ${ }^{9,10}$ which have broad anti-infective effects while meeting patient needs for relief of symptoms.

Antiseptics are a class of antimicrobial agent which kill via a physical action on the bacteria. ${ }^{11}$ In addition to bactericidal activity, some antiseptics - such as amylmetacresol (AMC) and 2,4-dichlorobenzyl alcohol (DCBA) - have been shown to have antiviral effects in vitro ${ }^{12,13}$ and anesthetic-like effects ${ }^{14}$ with established benefits in providing symptomatic relief of pain. ${ }^{15,16}$

Bacterial infections contribute to $5 \%-15 \%$ of pharyngitis cases in adults. ${ }^{4,17,18}$ The most common bacterial cause of acute pharyngitis, and the reason for legitimate antibiotic prescribing to prevent complications, is group A $\beta$-hemolytic Streptococcus (GABHS or Streptococcus pyogenes). ${ }^{3,19}$ It is responsible for $\sim 30 \%$ of cases in children ${ }^{20}$ and is less frequent in adults at $\sim 10 \%$ of cases, ${ }^{17}$ but rarely results in complications. ${ }^{3}$ A number of other bacteria have also been implicated in infections of the throat, which may present with a more complicated pathology or represent either opportunistic infection or an underlying medical condition.

Less common species recovered from patients presenting with symptoms of pharyngitis or with a clinical diagnosis of pharyngitis include Fusobacterium necrophorum, ${ }^{21}$ described in a recent study as a true pathogen rather than a colonizer of the oropharynx, ${ }^{22}$ and the Streptococcus dysgalactiae subspecies equisimilis, which can cause severe or recurrent pharyngitis, ${ }^{3,17,23}$ although there is insufficient evidence of a role for S. dysgalactiae in other adverse outcomes. ${ }^{3}$ Moraxella catarrhalis has been frequently isolated from patients with pharyngitis in combination with $S$. pyogenes, ${ }^{24}$ which may be significant considering that separate studies have demonstrated that $M$. catarrhalis potentiates the adhesion of $S$. pyogenes to the nasopharyngeal epithelium. ${ }^{25,26}$ Other bacteria cultured from patients with pharyngitis include Haemophilus influenza, ${ }^{27}$ Arcanobacterium haemolyticum ${ }^{28}$ and the opportunistic pathogen, Staphylococcus aureus, although the clinical significance of $S$. aureus association is not known. ${ }^{19}$

In patients diagnosed with tonsillitis, F. necrophorum, appears to be a clinically important species, with a prevalence significantly higher in subjects with clinical tonsillitis compared to subjects without tonsillitis. ${ }^{29} \mathrm{~S}$. aureus has also been identified as a common cause of tonsillitis ${ }^{30,31}$ and was the most common pathogen isolated from patients undergoing tonsillectomy due to recurrent tonsillitis. ${ }^{30} \mathrm{H}$. influenza has similarly been recovered from patients with tonsillitis, although the clinical significance is currently unknown. ${ }^{27}$

Non-antibiotic antimicrobial treatments could potentially benefit patients with bacterial pharyngitis by offering not only antimicrobial activity but also symptomatic relief. The in vitro activity of 10 lozenge formulations has previously been investigated against $S$. pyogenes and $S$. aureus. ${ }^{32}$ In this study, the in vitro bactericidal activity of AMC/DCBA lozenges against a broader range of potentially pathogenic oropharyngeal bacteria was assessed to evaluate the potential in vivo action of these lozenges against organisms associated with pharyngitis.

\section{Methods and materials Test samples}

For the bactericidal assay, AMC $0.6 \mathrm{mg}$, DCBA $1.2 \mathrm{mg}$ lozenges (Strepsils Honey and Lemon, Reckitt Benckiser Healthcare Ltd, Slough, UK) were dissolved into $5 \mathrm{~mL}$ of artificial saliva medium $(0.1 \%$ meat extract [VWR International, Lutterworth, UK], $0.2 \%$ yeast extract [VWR International], $0.5 \%$ protease peptone [Oxoid, Basingstoke, UK], 0.02\% potassium chloride [Fisher Scientific, Loughborough, UK], 0.02\% sodium chloride [Fisher Scientific], 0.03\% calcium carbonate [Fisher Scientific], 0.2\% glucose [VWR International], 0.2\% mucin from porcine stomach Type II [Sigma Aldrich, Gillingham, Dorset, UK], $\mathrm{pH} 6.7 \pm 0.3$ ) at $44^{\circ} \mathrm{C} \pm 1^{\circ} \mathrm{C}$. 


\section{Test organisms and incubations}

S. aureus (NCTC7445, Public Health England, Salisbury, UK) were cultured on tryptone soya agar (SGL, Corby, UK) at $32^{\circ} \mathrm{C} \pm 2{ }^{\circ} \mathrm{C} ;$ S. pyogenes (NCTC12696, Public Health England) were cultured on Columbia blood agar with 5\% defibrinated sheep blood (SGL) at $36^{\circ} \mathrm{C} \pm 2{ }^{\circ} \mathrm{C} ;$ M. catarrhalis (NCTC3622, Public Health England) were cultured on Columbia blood agar (SGL) at $32^{\circ} \mathrm{C} \pm 2{ }^{\circ} \mathrm{C} ; \mathrm{H}$. influenza (NCTC4842, Public Health England) were cultured on chocolate blood agar (SGL) at $32^{\circ} \mathrm{C} \pm 2{ }^{\circ} \mathrm{C}$; F. necrophorum (NCTC12238, Public Health England) were cultured on anaerobic blood agar (FAA) with 5\% horse blood (SGL) at $37^{\circ} \mathrm{C} \pm 2^{\circ} \mathrm{C}$ anaerobically; . haemolyticum (NCIMB702294, NCIMB, Aberdeen, UK) were cultured on Columbia blood agar with $5 \%$ defibrinated sheep blood at $36^{\circ} \mathrm{C} \pm 2^{\circ} \mathrm{C} ; S$. dysgalactiae (ATCC12388, LGC, Teddington, UK) were cultured on Columbia blood agar with $5 \%$ defibrinated sheep blood at $36^{\circ} \mathrm{C} \pm 2^{\circ} \mathrm{C}$.

\section{Bactericidal assay}

The bactericidal assay was performed following a protocol similar to the Clinical and Laboratory Standards Institute approved guideline. ${ }^{33}$ Specifically, inoculum cultures were prepared for each challenge organism to give an approximate population of $10^{8}$ colony-forming unit (CFU)/mL in saline ( $0.9 \%$ sodium chloride [Fisher Scientific]). One inoculum suspension was prepared for each replicate tested. Test sample $(4.9 \mathrm{~mL})$ was prepared as above and inoculated with 0.1 $\mathrm{mL}$ of the inoculum suspension. The solution was vortexed thoroughly to mix and then tested after 1-, 5- and 10-minute contact times, consistent with the time a lozenge takes to dissolve in the mouth, ${ }^{16}$ by removing $1 \mathrm{~mL}$ of sample/inocula mixture and transferring into $9 \mathrm{~mL}$ of neutralizing diluent $(0.1 \%$ peptone water [VWR International], $0.9 \%$ sodium chloride [Fisher Scientific], 0.3\% lecithin [MP Biomedicals, Illkirch-Graffenstaden, France], 1\% polysorbate 80 [Univar, Bradford, UK], $\mathrm{pH}$ 6.6 \pm 0.2 ). Neutralization validation was carried out against all test organisms. Solutions were serially diluted to $10^{-5}$, plated onto the appropriate agar medium and incubated for a minimum of 3 days. A positive control sample of $4.9 \mathrm{~mL}$ artificial saliva medium and $0.1 \mathrm{~mL}$ of the test inoculum for each organism was also prepared without exposure to test samples and assayed at a 30-minute time point. Test control counts were performed to confirm the total population of the culture suspensions used for each test replicate. The test controls were used to calculate the log reduction on exposure to test samples. Mean log reduction in CFUs per milliliter was calculated from three test replicates.

\section{Results}

\section{In vitro bactericidal activity of AMC/ DCBA lozenges}

For all test organisms, evidence of bactericidal activity was recorded at the 1-minute time point (Table 1, Figure 1), and test control counts demonstrated that the test method and media did not affect the survival of the organisms. For S. pyogenes, $H$. influenza, A. haemolyticum and $F$. necrophorum, the decrease in $\mathrm{CFU} / \mathrm{mL}$ at 1 minute exceeded 3 $\log _{10}\left(99.9 \%\right.$ decrease), whereas greater than $3 \log _{10}$ reductions were recorded at 5 minutes for $S$. dysgalactiae and M. catarrhalis and at 10 minutes for $S$. aureus. Additionally, at all time points, the SD (Table 1) of the replicates was small $\left(\leq 0.9 \log _{10} \mathrm{CFU} / \mathrm{mL}\right)$, indicating consistent and reproducible observations.

\section{Discussion}

This study examined the bactericidal action of an antiseptic lozenge containing AMC and DCBA. The organisms tested included gram-positive cocci (S. pyogenes, S. aureus, S. dysgalactiae) and bacilli (A. haemolyticum), as well as gramnegative cocci (M. catarrhalis) and bacilli (H. influenza, F. necrophorum), representing a broad range of bacterial cell structures and sensitivities.

The results demonstrated that the AMC/DCBA lozenge exhibits broad bactericidal activity against a range of organisms implicated in pharyngitis and the rapid activity observed is consistent with the time taken for a lozenge to dissolve in the mouth. ${ }^{16}$

For all test organisms, evidence of bactericidal activity for the AMC/DCBA lozenge was recorded at the 1-minute time point. Of particular interest is the robust bactericidal activity against $S$. pyogenes, the most frequent cause of bacterial pharyngitis. ${ }^{4}$ Reductions exceeding $99.9 \%$ were achieved by 1 minute for $S$. pyogenes, $H$. influenza, A. haemolyticum and $F$. necrophorum, by 5 minutes for $S$. dysgalactiae and M. catarrhalis and by 10 minutes for $S$. aureus. The bactericidal activity of an AMC/DCBA lozenge within a 10-minute period is important as it is consistent with the duration that a lozenge remains in the mouth; furthermore, the active ingredients were also tested at the expected concentration achieved when a lozenge is dissolved in the mouth, assuming a volume of $5 \mathrm{~mL}$ of saliva.

A previous in vitro evaluation of the bactericidal activity of antiseptic lozenges ([DCBA $1.2 \mathrm{mg}$, menthol $8 \mathrm{mg}$, AMC 0.6 $\mathrm{mg}$ ] and [DCBA $1.2 \mathrm{mg}$, AMC $0.6 \mathrm{mg}$ ]) against $S$. pyogenes and $S$. aureus demonstrated antibacterial effectiveness. ${ }^{32}$ Both $\mathrm{AMC}$ and DCBA formulations were highly active against the 


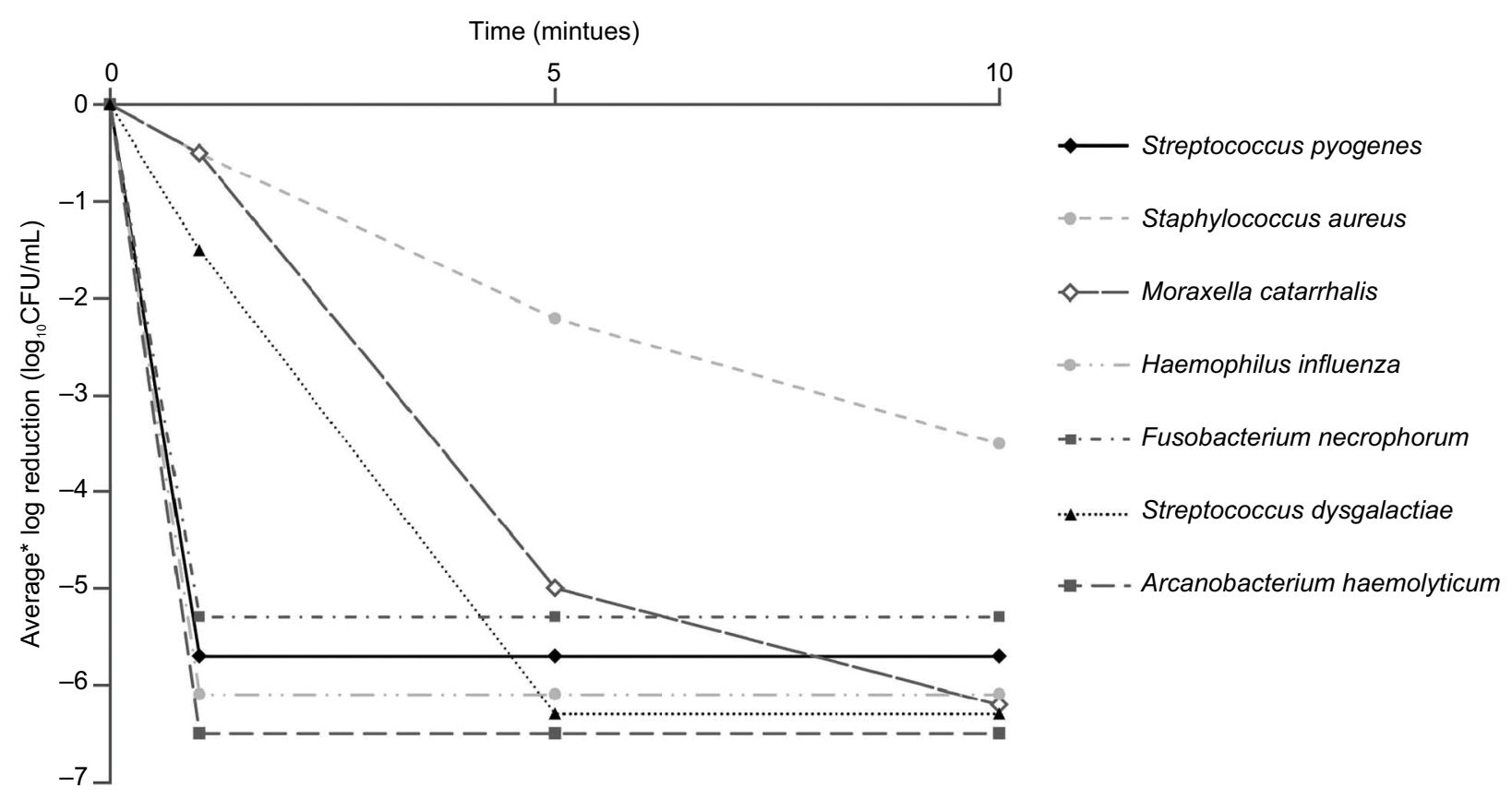

Figure I Bactericidal activity of an AMC/DCBA lozenge.

Notes: Bactericidal activity was measured against seven common oropharyngeal organisms over a 10 -minute period. Bactericidal activity was defined as a decrease in bacterial count (CFUs per $\mathrm{mL}$ ), using the average of three test replicates. *Average of the three test replicates.

Abbreviations: AMC, amylmetacresol; CFUs, colony-forming units; DCBA, 2,4-dichlorobenzyl alcohol.

Table I Control and test counts of challenge organisms

\begin{tabular}{|c|c|c|c|c|}
\hline \multirow[t]{2}{*}{ Challenge organism } & \multirow{2}{*}{$\begin{array}{l}\text { Test control count } \\
\left(\text { mean } \log _{10} \text { CFUs/mL } \pm \text { SD) }\right.\end{array}$} & \multicolumn{3}{|c|}{ Log reduction (mean $\log _{10}$ CFUs/mL \pm SD) } \\
\hline & & I minute & 5 minutes & 10 minutes \\
\hline Staphylococcus aureus & $6.5 \pm 0.1$ & $0.5 \pm 0.2$ & $2.2 \pm 0.1$ & $3.5 \pm 0.1$ \\
\hline Streptococcus pyogenes & $6.7 \pm 0.1$ & $5.7 \pm 0.1$ & $5.7 \pm 0.1$ & $5.7 \pm 0.1$ \\
\hline Moraxella catarrhalis & $7.2 \pm 0.1$ & $0.5 \pm 0.1$ & $5.0 \pm 0.9$ & $6.2 \pm 0.1$ \\
\hline Haemophilus influenza & $7.1 \pm 0.1$ & $6.1 \pm 0.1$ & $6.0 \pm 0.1$ & $6.2 \pm 0.1$ \\
\hline Fusobacterium necrophorum & $6.3 \pm 0.0$ & $5.3 \pm 0.0$ & $5.3 \pm 0.0$ & $5.3 \pm 0.0$ \\
\hline Arcanobacterium haemolyticum & $7.5 \pm 0.0$ & $6.5 \pm 0.0$ & $6.5 \pm 0.0$ & $6.5 \pm 0.0$ \\
\hline Streptococcus dysgalactiae & $7.3 \pm 0.0$ & $1.5 \pm 0.2$ & $6.3 \pm 0.0$ & $6.3 \pm 0.0$ \\
\hline
\end{tabular}

Abbreviation: CFU, colony-forming unit.

bacteria tested within 5 minutes of exposure, in contrast to the slow and weak action of the local antibiotic tyrothricin. ${ }^{33}$ The data generated in this study support and expand upon these previously published observations, providing further evidence of effectiveness against a broader range of bacterial species under in vitro conditions, including those where knowledge of their clinical pathology in pharyngitis is continuing to evolve or those that represent either an opportunistic infection or an underlying medical condition. These data likewise complement recent studies showing the in vitro viricidal effects of lozenges containing AMC/DCBA (and the active ingredients as free substances) against parainfluenza virus type 3 , cytomegalovirus, respiratory syncytial virus, influenza
A and severe acute respiratory syndrome coronavirus. ${ }^{12,13}$ In addition to antimicrobial activity, AMC and DCBA are proven to provide relief from the symptoms of pharyngitis, particularly sore throat, likely through their demonstrated local anesthetic-like action against voltage-gated neuronal sodium channels, ${ }^{14,34}$ and therefore may benefit patients presenting with either bacterial or viral pharyngitis. Furthermore, by relieving symptoms and managing patient expectations, the number of instances of inappropriate antibiotic prescribing for viral pharyngitis may be reduced.

A limitation of this study is that these observations were performed in vitro and therefore do not fully reflect the environment of the throat. For example, the throat may 
contain multiple microorganisms whereas this study tested the bactericidal activity against organisms in isolation. The role of the patient's immune system and swallowing action on the antimicrobial activity of the lozenge or active ingredients can also not be determined using in vitro methodology. However, the incidence of these bacteria is relevantly low in the general population; therefore, studying the bactericidal activity of AMC/DCBA in vivo can be challenging. Consequently, an in vitro approach is advantageous allowing the rapid generation of robust data, for multiple organisms simultaneously, that can be used to evaluate the potential of AMC/DCBA for efficacy in vivo.

\section{Conclusion}

These data show that an AMC/DCBA lozenge demonstrates bactericidal activity against all test organisms, representing a broad range of bacterial cell structures, from 1 minute and achieves greater than $99.9 \%$ kill for all test organisms within 10 minutes, which is consistent with the duration that a lozenge remains in the mouth.

Therefore, patients with uncomplicated bacterial pharyngitis, including those taking antibiotics, from low-risk populations and without additional risk factors, may benefit from the antiseptic action of AMC/DCBA against a range of bacterial species associated with pharyngitis. Most cases of pharyngitis should not require antibiotics as they are typically self-limiting and often viral in origin. Therefore, over-the-counter antiseptics like AMC/DCBA may be more appropriate, unless the condition deteriorates or a streptococcal infection is diagnosed.

\section{Data sharing statement}

All data generated or analyzed during this study are included in this manuscript.

\section{Acknowledgments}

The authors would like to thank Aisat Fatade Ogunpola (a former employee of Reckitt Benckiser Healthcare Ltd, UK) for laboratory support. Medical writing assistance was provided by Daniel East at Elements Communications Ltd, Westerham, UK and was funded by Reckitt Benckiser Healthcare Ltd, UK. This work was supported by Reckitt Benckiser Healthcare Ltd, UK.

\section{Author contributions}

All authors contributed to data analysis, drafting or revising the article, gave final approval of the version to be published, and agree to be accountable for all aspects of the work.

\section{Disclosure}

Derek Matthews, Robert Atkinson and Adrian Shephard are employees of Reckitt Benckiser Healthcare Ltd, UK. The authors report no other conflicts of interest in this work.

\section{References}

1. Renner B, Mueller CA, Shephard A. Environmental and non-infectious factors in the aetiology of pharyngitis (sore throat). Inflamm Res. 2012;61(10):1041-1052.

2. Vincent MT, Celestin N, Hussain AN. Pharyngitis. Am Fam Physician. 2004;69(6):1465-1470.

3. ESCMID Sore Throat Guideline Group; Pelucchi C, Grigoryan L, et al. Guideline for the management of acute sore throat. Clin Microbiol Infect. 2012;18(Suppl 1):1-28.

4. Worrall GJ. Acute sore throat. Can Fam Physician. 2007;53(11): 1961-1962.

5. Spinks A, Glasziou PP, Del Mar CB. Antibiotics for sore throat Cochrane Database Syst Rev. 2013;(11):CD000023.

6. van Driel ML, De Sutter A, Deveugele M, et al. Are sore throat patients who hope for antibiotics actually asking for pain relief? Ann Fam Med. 2006;4(6):494-499.

7. Linder JA, Singer DE. Desire for antibiotics and antibiotic prescribing for adults with upper respiratory tract infections. J Gen Intern Med. 2003;18(10):795-801.

8. World Health Organization. Antimicrobial Resistance Fact Sheet. Available from: http://www.who.int/en/news-room/fact-sheets/detail/antimicrobialresistance. Published February 15, 2018. Accessed July 07, 2018.

9. Dekker AR, Verheij TJ, van der Velden AW. Inappropriate antibiotic prescription for respiratory tract indications: most prominent in adult patients. Fam Pract. 2015;32(4):401-407.

10. Gulliford MC, Dregan A, Moore MV, et al. Continued high rates of antibiotic prescribing to adults with respiratory tract infection: survey of 568 UK general practices. BMJ Open. 2014;4(10):e006245.

11. Sheldon AT Jr. Antiseptic "resistance": real or perceived threat? Clin Infect Dis. 2005;40(11):1650-1656.

12. Oxford JS, Lambkin R, Gibb I, Balasingam S, Chan C, Catchpole A. A throat lozenge containing amyl meta cresol and dichlorobenzyl alcohol has a direct virucidal effect on respiratory syncytial virus, influenza A and SARS-CoV. Antivir Chem Chemother. 2005;16(2):129-134.

13. Shephard A, Zybeshari S. Virucidal action of sore throat lozenges against respiratory viruses parainfluenza type 3 and cytomegalovirus. Antiviral Res. 2015;123:158-162.

14. Foadi N, de Oliveira RC, Buchholz V, et al. A combination of topical antiseptics for the treatment of sore throat blocks voltage-gated neuronal sodium channels. Naunyn Schmiedebergs Arch Pharmacol. 2014;387(10):991-1000.

15. McNally D, Simpson M, Morris C, Shephard A, Goulder M. Rapid relief of acute sore throat with AMC/DCBA throat lozenges: randomised controlled trial. Int J Clin Pract. 2010;64(2):194-207.

16. Wade AG, Morris C, Shephard A, Crawford GM, Goulder MA. A multicentre, randomised, double-blind, single-dose study assessing the efficacy of AMC/DCBA Warm lozenge or AMC/DCBA Cool lozenge in the relief of acute sore throat. BMC Fam Pract. 2011;12(1):6.

17. Shephard A, Smith G, Aspley S, Schachtel BP. Randomised, doubleblind, placebo-controlled studies on flurbiprofen $8.75 \mathrm{mg}$ lozenges in patients with/without group A or C streptococcal throat infection, with an assessment of clinicians' prediction of "strep throat". Int J Clin Pract. 2015;69(1):59-71.

18. Radkova E, Burova N, Bychkova V, Devito R. Efficacy of flurbiprofen $8.75 \mathrm{mg}$ delivered as a spray or lozenge in patients with sore throat due to upper respiratory tract infection: a randomized, non-inferiority trial in the Russian Federation. J Pain Res. 2017;10:1591-1600.

19. Dagnelie CF, Touw-Otten FW, Kuyvenhoven MM, Rozenberg-Arska MR, de Melker RA. Bacterial flora in patients presenting with sore throat in Dutch general practice. Fam Pract. 1993;10(4):371-377. 
20. Shaikh N, Leonard E, Martin JM. Prevalence of streptococcal pharyngitis and streptococcal carriage in children: a meta-analysis. Pediatrics. 2010;126(3):e557-e564.

21. Batty A, Wren MW. Prevalence of Fusobacterium necrophorum and other upper respiratory tract pathogens isolated from throat swabs. $\mathrm{Br}$ J Biomed Sci. 2005;62(2):66-70.

22. Eaton C, Swindells J. The significance and epidemiology of Fusobacterium necrophorum in sore throats. J Infect. 2014;69(2): 194-196.

23. Harrington AT, Clarridge JE 3rd. Impact of identification of Streptococcus dysgalactiae subspecies equisimilis from throat cultures in an adult population. Diagn Microbiol Infect Dis. 2013;76(1):20-23.

24. Gergova RT, Petrova G, Gergov S, Minchev P, Mitov I, Strateva T. Microbiological features of upper respiratory tract infections in Bulgarian children for the period 1998-2014. Balkan Med J. 2016;33(6): 675-680.

25. Lafontaine ER, Wall D, Vanlerberg SL, Donabedian H, Sledjeski DD. Moraxella catarrhalis coaggregates with Streptococcus pyogenes and modulates interactions of S. pyogenes with human epithelial cells. Infect Immun. 2004;72(11):6689-6693.

26. Verhaegh SJ, Flores AR, van Belkum A, Musser JM, Hays JP. Differential virulence gene expression of group A Streptococcus serotype M3 in response to co-culture with Moraxella catarrhalis. PLoS One. 2013;8(4):e62549.
27. Mihancea N. Frequency and distribution per species, biotypes, resistance to antibiotics and beta-lactamase production of the hemophils isolated from patients with respiratory diseases. Roum Arch Microbiol Immunol. 1998;57(2):125-137.

28. Carlson P, Renkonen OV, Kontiainen S. Arcanobacterium haemolyticum and streptococcal pharyngitis. Scand J Infect Dis. 1994;26(3):283-287.

29. Jensen A, Hansen TM, Bank S, Kristensen LH, Prag J. Fusobacterium necrophorum tonsillitis: an important cause of tonsillitis in adolescents and young adults. Clin Microbiol Infect. 2015;21(3):266.e1-e3.

30. Katkowska M, Garbacz K, Stromkowski J. Staphylococcus aureus isolated from tonsillectomized adult patients with recurrent tonsillitis. APMIS. 2017;125(1):46-51.

31. Zautner AE, Krause M, Stropahl G, et al. Intracellular persisting Staphylococcus aureus is the major pathogen in recurrent tonsillitis. PLoS One. 2010;5(3):e9452.

32. Richards RM, Xing DK. In vitro evaluation of the antimicrobial activities of selected lozenges. J Pharm Sci. 1993;82(12):1218-1220.

33. CLSI. Methods for Determining Bactericidal Activity of Antimicrobial Agents. Approved Guideline, CLSI Document M26-A. Wayne, PA: Clinical and Laboratory Standards Institute (CLSI); 1999.

34. Buchholz V, Leuwer M, Ahrens J, Foadi N, Krampfl K, Haeseler G. Topical antiseptics for the treatment of sore throat block voltage-gated neuronal sodium channels in a local anaesthetic-like manner. Naunyn Schmiedebergs Arch Pharmacol. 2009;380(2):161-168.
International Journal of General Medicine

\section{Publish your work in this journal}

The International Journal of General Medicine is an international, peer-reviewed open-access journal that focuses on general and internal medicine, pathogenesis, epidemiology, diagnosis, monitoring and treatment protocols. The journal is characterized by the rapid reporting of reviews, original research and clinical studies across all disease areas.

\section{Dovepress}

The manuscript management system is completely online and includes a very quick and fair peer-review system, which is all easy to use. Visit http://www.dovepress.com/testimonials.php to read real quotes from published authors. 